\title{
Positive Solutions for Systems of Coupled Fractional Boundary Value Problems
}

\author{
Johnny Henderson ${ }^{1}$, Rodica Luca ${ }^{2}$, Alexandru Tudorache ${ }^{3}$ \\ ${ }^{1}$ Department of Mathematics, Baylor University, Waco, Texas, USA \\ ${ }^{2}$ Department of Mathematics, Gh. Asachi Technical University, lasi, Romania \\ ${ }^{3}$ Faculty of Computer Engineering and Automatic Control, Gh. Asachi Technical University, lasi, Romania \\ Email: Johnny Henderson@baylor.edu, rluca@math.tuiasi.ro, alexandru.tudorache93@gmail.com
}

Received 21 September 2015; accepted 24 October 2015; published 27 October 2015

Copyright (C) 2015 by authors and Scientific Research Publishing Inc.

This work is licensed under the Creative Commons Attribution International License (CC BY).

http://creativecommons.org/licenses/by/4.0/

\section{(c) (i) Open Access}

\begin{abstract}
We investigate the existence and nonexistence of positive solutions for a system of nonlinear Riemann-Liouville fractional differential equations with coupled integral boundary conditions which contain some positive constants.
\end{abstract}

\section{Keywords}

\section{Riemann-Liouville Fractional Differential Equations, Coupled Integral Boundary Conditions, Positive Solutions}

\section{Introduction}

Fractional differential equations describe many phenomena in various fields of engineering and scientific disciplines such as physics, biophysics, chemistry, biology (such as blood flow phenomena), economics, control theory, signal and image processing, aerodynamics, viscoelasticity, electromagnetics, and so on (see [1]-[6]). For some recent developments on the topic, which can be seen in [7]-[19] and the references therein.

In this paper, we consider the system of nonlinear ordinary fractional differential equations

$$
\text { (S) }\left\{\begin{array}{l}
D_{0+}^{\alpha} u(t)+a(t) f(v(t))=0, t \in(0,1), \\
D_{0+}^{\beta} v(t)+b(t) g(u(t))=0, t \in(0,1),
\end{array}\right.
$$

with the coupled integral boundary conditions

$$
(B C)\left\{\begin{array}{l}
u(0)=u^{\prime}(0)=\cdots=u^{(n-2)}(0)=0, u(1)=\int_{0}^{1} v(s) \mathrm{d} H(s)+a_{0}, \\
v(0)=v^{\prime}(0)=\cdots=v^{(m-2)}(0)=0, v(1)=\int_{0}^{1} u(s) \mathrm{d} K(s)+b_{0},
\end{array}\right.
$$

How to cite this paper: Henderson, J., Luca, R. and Tudorache, A. (2015) Positive Solutions for Systems of Coupled Fractional Boundary Value Problems. Open Journal of Applied Sciences, 5, 600-608. 
where $n-1<\alpha \leq n, \quad m-1<\beta \leq m, \quad n, m \in \mathbb{N}, \quad n, m \geq 3, \quad D_{0+}^{\alpha}$ and $D_{0+}^{\beta}$ denote the Riemann-Liouville derivatives of orders $\alpha$ and $\beta$, respectively, the integrals from (BC) are Riemann-Stieltjes integrals, $a_{0}$ and $b_{0}$ are positive constants.

Under some assumptions on the functions $f$ and $g$, we shall prove the existence of positive solutions of problem $(S)$-(BC). By a positive solution of $(S)-(B C)$, we mean a pair of functions $(u, v) \in C\left([0,1] ; \mathbb{R}_{+}\right) \times C\left([0,1] ; \mathbb{R}_{+}\right)$ satisfying $(S)$ and $(B C)$ with $u(t)>0, v(t)>0$ for all $t \in(0,1]$. We shall also give sufficient conditions for the nonexistence of positive solutions for this problem. Some systems of fractional equations with parameters subject to coupled integral boundary conditions were studied in [20] by using the Guo-Krasnosel'skii fixed point theorem. We also mentioned the paper [21], where we investigated the existence and multiplicity of positive solutions for the system $D_{0+}^{\alpha} u(t)+f(t, v(t))=0, t \in(0,1), D_{0+}^{\beta} v(t)+g(t, u(t))=0, t \in(0,1)$, with the integral boundary conditions $(B C)$ with $a_{0}=b_{0}=0$ by using some theorems from the fixed point index theory and the Guo-Krasnosel'skii fixed point theorem. In [21], the nonlinearities $f$ and $g$ may be nonsingular or singular in $t=0$ and/or $t=1$. Some systems of Riemann-Liouville fractional equations with or without parameters subject to uncoupled boundary conditions are studied in the papers [22]-[25], and the book [26].

In Section 2, we present some auxiliary results which investigate a system of Riemann-Liouville fractional equations subject to coupled integral boundary conditions. In Section 3, we prove our main results, and an example which supports the obtained results is finally presented in Section 4. In the proof of our existence result, we shall use the Schauder fixed point theorem which we present now.

Theorem 1. Let $X$ be a Banach space and $Y \subset X$ a nonempty, bounded, convex and closed subset. If the operator $A: Y \rightarrow Y$ is completely continuous, then $A$ has at least one fixed point.

\section{Auxiliary Results}

We present here the definitions of the fractional integral and Riemann-Liouville fractional derivative of a function, and some auxiliary results from [20] and [22] that will be used to prove our main theorems.

Definition 2.1: The (left-sided) fractional integral of order $\alpha>0$ of a function $f:(0, \infty) \rightarrow \mathbb{R}$ is given by

$$
\left(I_{0+}^{\alpha} f\right)(t)=\frac{1}{\Gamma(\alpha)} \int_{0}^{t}(t-s)^{\alpha-1} f(s) \mathrm{d} s, t>0
$$

provided the right-hand side is pointwise defined on $(0, \infty)$, where $\Gamma(\alpha)$ is the Euler gamma function defined by $\Gamma(\alpha)=\int_{0}^{\infty} t^{\alpha-1} \mathrm{e}^{-t} \mathrm{~d} t, \alpha>0$.

Definition 2.2: The Riemann-Liouville fractional derivative of order $\alpha \geq 0$ for a function $f:(0, \infty) \rightarrow \mathbb{R}$ is given by

$$
\left(D_{0+}^{\alpha} f\right)(t)=\left(\frac{\mathrm{d}}{\mathrm{d} t}\right)^{n}\left(I_{0+}^{n-\alpha} f\right)(t)=\frac{1}{\Gamma(n-\alpha)}\left(\frac{\mathrm{d}}{\mathrm{d} t}\right)^{n} \int_{0}^{t} \frac{f(s)}{(t-s)^{\alpha-n+1}} \mathrm{~d} s, t>0,
$$

where $n=\llbracket \alpha \rrbracket+1$, provided that the right-hand side is pointwise defined on $(0, \infty)$.

The notation $\llbracket \alpha \rrbracket$ stands for the largest integer not greater than $\alpha$. If $\alpha=m \in \mathbb{N}$ then $D_{0+}^{m} f(t)=f^{(m)}(t)$ for $t>0$, and if $\alpha=0$ then $D_{0+}^{0} f(t)=f(t)$ for $t>0$.

We consider now the fractional differential system

$$
\left\{\begin{array}{l}
D_{0+}^{\alpha} u(t)+x(t)=0, t \in(0,1), n-1<\alpha \leq n, \\
D_{0+}^{\beta} v(t)+y(t)=0, t \in(0,1), m-1<\beta \leq m,
\end{array}\right.
$$

with the coupled integral boundary conditions

$$
\left\{\begin{array}{l}
u(0)=u^{\prime}(0)=\cdots=u^{(n-2)}(0)=0, u(1)=\int_{0}^{1} v(s) \mathrm{d} H(s), \\
v(0)=v^{\prime}(0)=\cdots=v^{(m-2)}(0)=0, v(1)=\int_{0}^{1} u(s) \mathrm{d} K(s),
\end{array}\right.
$$


where $n, m \in \mathbb{N}, n, m \geq 3$ and $H, K:[0,1] \rightarrow \mathbb{R}$ are functions of bounded variation.

Lemma 1. ([20]) If $H, K:[0,1] \rightarrow \mathbb{R}$ are functions of bounded variations,

$\Delta=1-\left(\int_{0}^{1} \tau^{\alpha-1} \mathrm{~d} K(\tau)\right)\left(\int_{0}^{1} \tau^{\beta-1} \mathrm{~d} H(\tau)\right) \neq 0$ and $x, y \in C(0,1) \cap L^{1}(0,1)$, then the unique solution of problem (1)-(2) is given by

$$
\left\{\begin{array}{l}
u(t)=\int_{0}^{1} G_{1}(t, s) x(s) \mathrm{d} s+\int_{0}^{1} G_{2}(t, s) y(s) \mathrm{d} s, \\
v(t)=\int_{0}^{1} G_{3}(t, s) y(s) \mathrm{d} s+\int_{0}^{1} G_{4}(t, s) x(s) \mathrm{d} s, t \in[0,1],
\end{array}\right.
$$

where

$$
\begin{aligned}
& G_{1}(t, s)=g_{1}(t, s)+\frac{t^{\alpha-1}}{\Delta}\left(\int_{0}^{1} \tau^{\beta-1} \mathrm{~d} H(\tau)\right)\left(\int_{0}^{1} g_{1}(\tau, s) \mathrm{d} K(\tau)\right), \\
& G_{2}(t, s)=\frac{t^{\alpha-1}}{\Delta} \int_{0}^{1} g_{2}(\tau, s) \mathrm{d} H(\tau), \\
& G_{3}(t, s)=g_{2}(t, s)+\frac{t^{\beta-1}}{\Delta}\left(\int_{0}^{1} \tau^{\alpha-1} \mathrm{~d} K(\tau)\right)\left(\int_{0}^{1} g_{2}(\tau, s) \mathrm{d} H(\tau)\right), \\
& G_{4}(t, s)=\frac{t^{\beta-1}}{\Delta} \int_{0}^{1} g_{1}(\tau, s) \mathrm{d} K(\tau), \forall t, s \in[0,1],
\end{aligned}
$$

and

$$
\left\{\begin{array}{l}
g_{1}(t, s)=\frac{1}{\Gamma(\alpha)}\left\{\begin{array}{l}
t^{\alpha-1}(1-s)^{\alpha-1}-(t-s)^{\alpha-1}, 0 \leq s \leq t \leq 1, \\
t^{\alpha-1}(1-s)^{\alpha-1}, 0 \leq t \leq s \leq 1,
\end{array}\right. \\
g_{2}(t, s)=\frac{1}{\Gamma(\beta)}\left\{\begin{array}{l}
t^{\beta-1}(1-s)^{\beta-1}-(t-s)^{\beta-1}, 0 \leq s \leq t \leq 1, \\
t^{\beta-1}(1-s)^{\beta-1}, 0 \leq t \leq s \leq 1 .
\end{array}\right.
\end{array}\right.
$$

Lemma 2. ([22]) The functions $g_{1}$ and $g_{2}$ given by (5) have the properties

a) $g_{1}, g_{2}:[0,1] \times[0,1] \rightarrow \mathbb{R}_{+}$are continuous functions and $g_{1}(t, s)>0, \quad g_{2}(t, s)>0$ for all $(t, s) \in(0,1) \times(0,1)$;

b) $g_{1}(t, s) \leq g_{1}\left(\theta_{1}(s), s\right), g_{2}(t, s) \leq g_{2}\left(\theta_{2}(s), s\right)$, for all $(t, s) \in[0,1] \times[0,1]$;

c) For any $c \in(0,1 / 2)$, we have

$$
\min _{t \in[c, 1-c]} g_{1}(t, s) \geq \gamma_{1} g_{1}\left(\theta_{1}(s), s\right), \min _{t \in[c, 1-c]} g_{2}(t, s) \geq \gamma_{2} g_{2}\left(\theta_{2}(s), s\right),
$$

for all $s \in[0,1]$, where $\gamma_{1}=c^{\alpha-1}, \quad \gamma_{2}=c^{\beta-1}, \quad \theta_{1}(s)=\left\{\begin{array}{ll}\frac{s}{1-(1-s)^{\frac{\alpha-1}{\alpha-2}}}, & s \in(0,1] \text {, } \\ \frac{\alpha-2}{\alpha-1}, & s=0,\end{array}\right.$ and $\theta_{2}(s)= \begin{cases}\frac{s}{1-(1-s)^{\frac{\beta-1}{\beta-2}}}, & s \in(0,1], \\ \frac{\beta-2}{\beta-1}, & s=0 .\end{cases}$

Lemma 3. ([20]) If $H, K:[0,1] \rightarrow \mathbb{R}$ are nondecreasing functions, and $\Delta>0$, then $G_{i}, i=1, \cdots, 4$, given by (4) are continuous functions on $[0,1] \times[0,1]$ and satisfy $G_{i}(t, s) \geq 0$ for all $(t, s) \in[0,1] \times[0,1], i=1, \cdots, 4$. Moreover, if $x, y \in C(0,1) \cap L^{1}(0,1)$ satisfy $x(t) \geq 0, y(t) \geq 0$ for all $t \in(0,1)$, then the solution $(u, v)$ of 
problem (1)-(2) satisfies $u(t) \geq 0, v(t) \geq 0$ for all $t \in[0,1]$.

Lemma 4. ([20]) Assume that $H, K:[0,1] \rightarrow \mathbb{R}$ are nondecreasing functions and $\Delta>0$. Then the functions $G_{i}, i=1, \cdots, 4$, satisfy the inequalities

$\left.a_{1}\right) G_{1}(t, s) \leq J_{1}(s), \forall(t, s) \in[0,1] \times[0,1]$, where

$$
J_{1}(s)=g_{1}\left(\theta_{1}(s), s\right)+\frac{1}{\Delta}\left(\int_{0}^{1} \tau^{\beta-1} \mathrm{~d} H(\tau)\right)\left(\int_{0}^{1} g_{1}(\tau, s) \mathrm{d} K(\tau)\right) ;
$$

$a_{2}$ ) For every $c \in(0,1 / 2)$, we have

$$
\min _{t \in[c, 1-c]} G_{1}(t, s) \geq \gamma_{1} J_{1}(s) \geq \gamma_{1} G_{1}\left(t^{\prime}, s\right), \forall t^{\prime}, s \in[0,1] ;
$$

$\left.b_{1}\right) G_{2}(t, s) \leq J_{2}(s), \forall(t, s) \in[0,1] \times[0,1]$, where $J_{2}(s)=\frac{1}{\Delta} \int_{0}^{1} g_{2}(\tau, s) \mathrm{d} H(\tau)$;

$b_{2}$ ) For every $c \in(0,1 / 2)$, we have

$$
\min _{t \in[c, 1-c]} G_{2}(t, s) \geq \gamma_{1} J_{2}(s) \geq \gamma_{1} G_{2}\left(t^{\prime}, s\right), \forall t^{\prime}, s \in[0,1] ;
$$

$\left.c_{1}\right) \quad G_{3}(t, s) \leq J_{3}(s), \forall(t, s) \in[0,1] \times[0,1]$, where

$$
J_{3}(s)=g_{2}\left(\theta_{2}(s), s\right)+\frac{1}{\Delta}\left(\int_{0}^{1} \tau^{\alpha-1} \mathrm{~d} K(\tau)\right)\left(\int_{0}^{1} g_{2}(\tau, s) \mathrm{d} H(\tau)\right) ;
$$

$c_{2}$ ) For every $c \in(0,1 / 2)$, we have

$$
\min _{t \in[c, 1-c]} G_{3}(t, s) \geq \gamma_{2} J_{3}(s) \geq \gamma_{2} G_{3}\left(t^{\prime}, s\right), \forall t^{\prime}, s \in[0,1] ;
$$

$\left.d_{1}\right) G_{4}(t, s) \leq J_{4}(s), \forall(t, s) \in[0,1] \times[0,1]$, where $J_{4} s=\frac{1}{\Delta} \int_{0}^{1} g_{1}(\tau, s) \mathrm{d} K(\tau)$;

$d_{2}$ ) For every $c \in(0,1 / 2)$, we have

$$
\min _{t \in[c, 1-c]} G_{4}(t, s) \geq \gamma_{2} J_{4}(s) \geq \gamma_{2} G_{4}\left(t^{\prime}, s\right), \forall t^{\prime}, s \in[0,1] .
$$

Lemma 5. ([20]) Assume that $H, K:[0,1] \rightarrow \mathbb{R}$ are nondecreasing functions, $\Delta>0, \quad c \in(0,1 / 2)$ and $x, y \in C(0,1) \cap L^{1}(0,1), \quad x(t) \geq 0, \quad y(t) \geq 0$ for all $t \in(0,1)$. Then the solution $(u(t), v(t)), t \in[0,1]$ of problem (1)-(2) (given by (3)) satisfies the inequalities

$$
\inf _{t \in[c, 1-c]} u(t) \geq \gamma_{1} \sup _{t^{\prime} \in[0,1]} u\left(t^{\prime}\right), \inf _{t \in[c, 1-c]} v(t) \geq \gamma_{2} \sup _{t^{\prime} \in[0,1]} v\left(t^{\prime}\right) .
$$

\section{Main Results}

We present first the assumptions that we shall use in the sequel.

$\left(J_{1}\right) H, K:[0,1] \rightarrow \mathbb{R}$ are nondecreasing functions and $\Delta=1-\left(\int_{0}^{1} \tau^{\alpha-1} \mathrm{~d} K(\tau)\right)\left(\int_{0}^{1} \tau^{\beta-1} \mathrm{~d} H(\tau)\right)>0$.

$\left(J_{2}\right)$ The functions $a, b:[0,1] \rightarrow[0, \infty)$ are continuous and there exist $t_{1}, t_{2} \in(0,1)$ such that $a\left(t_{1}\right)>0$, $b\left(t_{2}\right)>0$.

$\left(J_{3}\right) f, g:[0, \infty) \rightarrow[0, \infty)$ are continuous functions and there exists $c_{0}>0$ such that

$$
f(u)<\frac{c_{0}}{L}, g(u)<\frac{c_{0}}{L} \text { for all } u \in\left[0, c_{0}\right]
$$

where $L=\max \left\{\int_{0}^{1} a(s) J_{1}(s) \mathrm{d} s+\int_{0}^{1} b(s) J_{2}(s) \mathrm{d} s, \int_{0}^{1} b(s) J_{3}(s) \mathrm{d} s+\int_{0}^{1} a(s) J_{4}(s) \mathrm{d} s\right\}$ and $J_{i}, i=1, \cdots, 4$ are defined in Lemma 4.

$\left(J_{4}\right)$ are continuous functions and satisfy the conditions 


$$
\lim _{u \rightarrow \infty} f(u) / u=\infty, \lim _{u \rightarrow \infty} g(u) / u=\infty .
$$

By assumption $\left(J_{2}\right)$ we deduce that $\int_{0}^{1} a(s) J_{1}(s) \mathrm{d} s>0, \int_{0}^{1} b(s) J_{2}(s) \mathrm{d} s>0, \int_{0}^{1} b(s) J_{3}(s) d s>0$ and $\int_{0}^{1} a(s) J_{4}(s) d s>0$, that is, the constant $L$ from $\left(J_{3}\right)$ is positive.

Our first theorem is the following existence result for problem $(S)-(B C)$.

Theorem 2. Assume that assumptions $\left(J_{1}\right)-\left(J_{3}\right)$ hold. Then problem $(S)-(B C)$ has at least one positive solution for $a_{0}>0$ and $b_{0}>0$ sufficiently small.

Proof. We consider the system of ordinary fractional differential equations

$$
\left\{\begin{array}{l}
D_{0+}^{\alpha} h(t)=0, t \in(0,1) \\
D_{0+}^{\beta} k(t)=0, t \in(0,1)
\end{array}\right.
$$

with the coupled integral boundary conditions

$$
\left\{\begin{array}{l}
h(0)=h^{\prime}(0)=\cdots=h^{(n-2)}(0)=0, h(1)=\int_{0}^{1} k(s) \mathrm{d} H(s)+a_{0} \\
k(0)=k^{\prime}(0)=\cdots=k^{(m-2)}(0)=0, k(1)=\int_{0}^{1} h(s) \mathrm{d} K(s)+b_{0}
\end{array}\right.
$$

with $a_{0}>0$ and $b_{0}>0$.

The above problem (6)-(7) has the solution

$$
\begin{aligned}
& h(t)=\frac{t^{\alpha-1}}{\Delta}\left(a_{0}+b_{0} \int_{0}^{1} s^{\beta-1} \mathrm{~d} H(s)\right), t \in[0,1], \\
& k(t)=\frac{t^{\beta-1}}{\Delta}\left(b_{0}+a_{0} \int_{0}^{1} s^{\alpha-1} \mathrm{~d} K(s)\right), t \in[0,1],
\end{aligned}
$$

where $\Delta$ is defined in $\left(J_{1}\right)$. By assumption $\left(J_{1}\right)$ we obtain $h(t)>0$ and $k(t)>0$ for all $t \in(0,1]$.

We define the functions $x(t)$ and $y(t), t \in[0,1]$ by

$$
x(t)=u(t)-h(t), y(t)=v(t)-k(t), \forall t \in[0,1],
$$

where $(u, v)$ is a solution of $(S)-(B C)$. Then $(S)-(B C)$ can be equivalently written as

$$
\left\{\begin{array}{l}
D_{0+}^{\alpha} x(t)+a(t) f(y(t)+k(t))=0, t \in(0,1) \\
D_{0+}^{\beta} y(t)+b(t) g(x(t)+h(t))=0, t \in(0,1)
\end{array}\right.
$$

with the boundary conditions

$$
\left\{\begin{array}{l}
x(0)=x^{\prime}(0)=\cdots=x^{(n-2)}(0)=0, x(1)=\int_{0}^{1} y(s) \mathrm{d} H(s), \\
y(0)=y^{\prime}(0)=\cdots=y^{(m-2)}(0)=0, y(1)=\int_{0}^{1} x(s) \mathrm{d} K(s) .
\end{array}\right.
$$

Using the Green's functions $G_{i}, i=1, \cdots, 4$, from Lemma 1 , a pair $(x, y)$ is a solution of problem (9)-(10) if and only if $(x, y)$ is a solution for the nonlinear integral equations

$$
\left\{\begin{array}{l}
x(t)=\int_{0}^{1} G_{1}(t, s) a(s) f(y(s)+k(s)) \mathrm{d} s+\int_{0}^{1} G_{2}(t, s) b(s) g(x(s)+h(s)) \mathrm{d} s, t \in[0,1], \\
y(t)=\int_{0}^{1} G_{3}(t, s) b(s) g(x(s)+h(s)) \mathrm{d} s+\int_{0}^{1} G_{4}(t, s) a(s) f(y(s)+k(s)) \mathrm{d} s, t \in[0,1],
\end{array}\right.
$$

where $h(t)$ and $k(t), t \in[0,1]$ are given in (8).

We consider the Banach space $X=C([0,1])$ with the supremum norm $\|\cdot\|$, the space $Y=X \times X$ with the norm $\|(x, y)\|_{Y}=\|x\|+\|y\|$, and we define the set

$$
E=\left\{x \in C([0,1]), 0 \leq x(t) \leq c_{0}, \forall t \in[0,1]\right\} \subset X .
$$

We also define the operators $S_{1}, S_{2}: E \times E \rightarrow X$ and $S: E \times E \rightarrow Y$ by 


$$
\begin{aligned}
& S_{1}(x, y)(t)=\int_{0}^{1} G_{1}(t, s) a(s) f(y(s)+k(s)) \mathrm{d} s+\int_{0}^{1} G_{2}(t, s) b(s) g(x(s)+h(s)) \mathrm{d} s, \\
& S_{2}(x, y)(t)=\int_{0}^{1} G_{3}(t, s) b(s) g(x(s)+h(s)) \mathrm{d} s+\int_{0}^{1} G_{4}(t, s) a(s) f(y(s)+k(s)) \mathrm{d} s,
\end{aligned}
$$

for all $t \in[0,1]$, and $S(x, y)=\left(S_{1}(x, y), S_{2}(x, y)\right),(x, y) \in E \times E$.

For sufficiently small $a_{0}>0$ and $b_{0}>0$, by (J3), we deduce

$$
f(y(t)+k(t)) \leq \frac{c_{0}}{L}, g(x(t)+h(t)) \leq \frac{c_{0}}{L}, \forall t \in[0,1], \forall x, y \in E .
$$

Then, by using Lemma 3, we obtain $S_{1}(x, y)(t) \geq 0, S_{2}(x, y)(t) \geq 0$ for all $t \in[0,1]$ and $(x, y) \in E \times E$. By Lemma 4, for all $(x, y) \in E \times E$, we have

$$
\begin{aligned}
& S_{1}(x, y)(t) \leq \int_{0}^{1} J_{1}(s) a(s) f(y(s)+k(s)) \mathrm{d} s+\int_{0}^{1} J_{2}(s) b(s) g(x(s)+h(s)) \mathrm{d} s \\
& \leq \frac{c_{0}}{L}\left(\int_{0}^{1} a(s) J_{1}(s) \mathrm{d} s+\int_{0}^{1} b(s) J_{2}(s) \mathrm{d} s\right) \leq c_{0}, \forall t \in[0,1],
\end{aligned}
$$

and

$$
\begin{aligned}
& S_{2}(x, y)(t) \leq \int_{0}^{1} J_{3}(s) b(s) g(x(s)+h(s)) \mathrm{d} s+\int_{0}^{1} J_{4}(s) a(s) f(y(s)+k(s)) \mathrm{d} s \\
& \leq \frac{c_{0}}{L}\left(\int_{0}^{1} b(s) J_{3}(s) \mathrm{d} s+\int_{0}^{1} a(s) J_{4}(s) \mathrm{d} s\right) \leq c_{0}, \forall t \in[0,1] .
\end{aligned}
$$

Therefore $S(E \times E) \subset E \times E$.

Using standard arguments, we deduce that $S$ is completely continuous. By Theorem 1, we conclude that $S$ has a fixed point $(x, y) \in E \times E$, which represents a solution for problem (9)-(10). This shows that our problem (S)-(BC) has a positive solution $(u, v)$ with $u=x+h, v=y+k$ for sufficiently small $a_{0}>0$ and $b_{0}>0$.

In what follows, we present sufficient conditions for the nonexistence of positive solutions of $(S)-(B C)$.

Theorem 3. Assume that assumptions $\left(J_{1}\right),\left(J_{2}\right)$ and $\left(J_{4}\right)$ hold. Then problem $(S)-(B C)$ has no positive solution for $a_{0}$ and $b_{0}$ sufficiently large.

Proof. We suppose that $(u, v)$ is a positive solution of $(S)-(B C)$. Then $(x, y)$ with $x=u-h, y=v-k$ is a solution for problem (9)-(10), where $(h, k)$ is the solution of problem (6)-(7) (given by (8)). By $\left(J_{2}\right)$ there exists $c \in(0,1 / 2)$ such that $t_{1}, t_{2} \in(c, 1-c)$, and then $\int_{c}^{1-c} a(s) J_{1}(s) \mathrm{d} s>0, \int_{c}^{1-c} b(s) J_{2}(s) \mathrm{d} s>0$, $\int_{c}^{1-c} b(s) J_{3}(s) \mathrm{d} s>0, \int_{c}^{1-c} a(s) J_{4}(s) \mathrm{d} s>0$. Now by using Lemma 3, we have $x(t) \geq 0, y(t) \geq 0$ for all $t \in[0,1]$, and by Lemma 5 we obtain $\inf _{t \in[c, 1-c]} x(t) \geq \gamma_{1}\|x\|$ and $\inf _{t \in[c, 1-c]} y(t) \geq \gamma_{2}\|y\|$.

Using now (8), we deduce that $\inf _{t \in[c, 1-c]} h(t)=\gamma_{1}\|h\|$ and $\inf _{t \in[c, 1-c]} k(t)=\gamma_{2}\|k\|$. Therefore, we obtain $\inf _{t \in[c, 1-c]}(x(t)+h(t)) \geq \gamma_{1}\|x+h\|$ and $\inf _{t \in[c, 1-c]}(y(t)+k(t)) \geq \gamma_{2}\|y+k\|$.

We now consider $R=\left(\gamma_{1}^{2} \int_{c}^{1-c} b(s) J_{2}(s) \mathrm{d} s\right)^{-1}>0$. By using $\left(J_{4}\right)$, for $R$ defined above, we conclude that there exists $M>0$ such that $f(u)>2 R u, g(u)>2 R u$ for all $u \geq M$. We consider $a_{0}>0$ and $b_{0}>0$ sufficiently large such that $\inf _{t \in[c, 1-c]}(x(t)+h(t)) \geq M$ and $\inf _{t \in[c, 1-c]}(y(t)+k(t)) \geq M$. By (J $)$, (9), (10) and the above inequalities, we deduce that $\|x\|>0$ and $\|y\|>0$.

Now by using Lemma 4 and the above considerations, we have

$$
\begin{aligned}
x(c) & =\int_{0}^{1} G_{1}(c, s) a(s) f\left(y(s)+k(s) \mathrm{d} s+\int_{0}^{1} G_{2}(c, s) b(s) g(x(s)+h(s)) \mathrm{d} s\right. \\
& \geq \gamma_{1} \int_{0}^{1} J_{2}(s) b(s) g(x(s)+h(s)) \mathrm{d} s \geq \gamma_{1} \int_{c}^{1-c} J_{2}(s) b(s) g(x(s)+h(s)) \mathrm{d} s \\
& \geq 2 R \gamma_{1} \int_{c}^{1-c} J_{2}(s) b(s)(x(s)+h(s)) \mathrm{d} s \geq 2 R \gamma_{1} \int_{c}^{1-c} J_{2}(s) b(s) \inf _{\tau \in[c, 1-c]}(x(\tau)+h(\tau)) \mathrm{d} s \\
& \geq 2 R \gamma_{1}^{2} \int_{c}^{1-c} J_{2}(s) b(s)\|x+h\| \mathrm{d} s=2\|x+h\| \geq 2\|x\| .
\end{aligned}
$$


Therefore, we obtain $\|x\| \leq \frac{1}{2} x(c) \leq \frac{1}{2}\|x\|$, which is a contradiction, because $\|x\|>0$. Then, for $a_{0}$ and $b_{0}$ sufficiently large, our problem $(S)-(B C)$ has no positive solution.

\section{An Example}

We consider $a(t)=1, \quad b(t)=1$ for all $t \in[0,1], \quad \alpha=7 / 3 \quad(n=3), \quad \beta=5 / 2 \quad(m=3), H(t)=t^{2}$, for all $t \in[0,1], \quad K(t)=\left\{\begin{array}{l}0, t \in[0,1 / 3), \\ 1, t \in[1 / 3,2 / 3), \\ 3 / 2, t \in[2 / 3,1] .\end{array}\right.$ then $\int_{0}^{1} v(s) \mathrm{d} H(s)=2 \int_{0}^{1} s v(s) \mathrm{d} s \quad$ and $\quad \int_{0}^{1} u(s) \mathrm{d} K(s)=u\left(\frac{1}{3}\right)+\frac{1}{2} u\left(\frac{2}{3}\right)$. We also consider the functions $f, g:[0, \infty) \rightarrow[0, \infty), f(x)=\tilde{a} x^{2}, g(x)=\tilde{b} x^{3}$, for all $x \in[0, \infty)$, with $\tilde{a}, \tilde{b}>0$. We have $\lim _{x \rightarrow \infty} f(x) / x=\lim _{x \rightarrow \infty} g(x) / x=\infty$.

Therefore, we consider the system of fractional differential equations

$$
\left(S_{0}\right)\left\{\begin{array}{l}
D_{0+}^{7 / 3} u(t)+\tilde{a} v^{2}(t)=0, t \in(0,1) \\
D_{0+}^{5 / 2} v(t)+\tilde{b} u^{3}(t)=0, t \in(0,1)
\end{array}\right.
$$

with the boundary conditions

$$
\left(B C_{0}\right)\left\{\begin{array}{l}
u(0)=u^{\prime}(0)=0, u(1)=2 \int_{0}^{1} s v(s) \mathrm{d} s+a_{0}, \\
v(0)=v^{\prime}(0)=0, v(1)=u\left(\frac{1}{3}\right)+\frac{1}{2} u\left(\frac{2}{3}\right)+b_{0} .
\end{array}\right.
$$

Then we obtain

$$
\Delta=1-\left(\int_{0}^{1} \tau^{4 / 3} \mathrm{~d} K(\tau)\right)\left(\int_{0}^{1} \tau^{3 / 2} \mathrm{~d} H(\tau)\right)=1-\left(\left(\frac{1}{3}\right)^{4 / 3}+\frac{1}{2}\left(\frac{2}{3}\right)^{4 / 3}\right)\left(2 \int_{0}^{1} \tau^{5 / 2} \mathrm{~d} \tau\right)=\frac{21 \sqrt[3]{3}-4-4 \sqrt[3]{2}}{21 \sqrt[3]{3}} \approx 0.70153491>0
$$

We also deduce

$$
\begin{aligned}
& g_{1}(t, s)=\frac{1}{\Gamma(7 / 3)}\left\{\begin{array}{l}
t^{4 / 3}(1-s)^{4 / 3}-(t-s)^{4 / 3}, 0 \leq s \leq t \leq 1, \\
t^{4 / 3}(1-s)^{4 / 3}, 0 \leq t \leq s \leq 1,
\end{array}\right. \\
& g_{2}(t, s)=\frac{4}{3 \sqrt{\pi}}\left\{\begin{array}{l}
t^{3 / 2}(1-s)^{3 / 2}-(t-s)^{3 / 2}, 0 \leq s \leq t \leq 1, \\
t^{3 / 2}(1-s)^{3 / 2}, 0 \leq t \leq s \leq 1,
\end{array}\right.
\end{aligned}
$$

$\theta_{1}(s)=\frac{1}{4-6 s+4 s^{2}-s^{3}}, \theta_{2}(s)=\frac{1}{3-3 s+s^{2}}$ for all $s \in[0,1]$. For the functions $J_{i}, i=1, \cdots, 4$, we obtain

$$
J_{1}(s)=\left\{\begin{array}{l}
\frac{1}{\Gamma(7 / 3)}\left\{\frac{s(1-s)^{4 / 3}}{\left(4-6 s+4 s^{2}-s^{3}\right)^{1 / 3}}+\frac{2}{21 \sqrt[3]{3} \Delta}\left[2(1-s)^{4 / 3}-2(1-3 s)^{4 / 3}+(2-2 s)^{4 / 3}-(2-3 s)^{4 / 3}\right]\right\}, 0 \leq s<1 / 3, \\
\frac{1}{\Gamma(7 / 3)}\left\{\frac{s(1-s)^{4 / 3}}{\left(4-6 s+4 s^{2}-s^{3}\right)^{1 / 3}}+\frac{2}{21 \sqrt[3]{3} \Delta}\left[2(1-s)^{4 / 3}+(2-2 s)^{4 / 3}-(2-3 s)^{4 / 3}\right]\right\}, 1 / 3 \leq s<2 / 3, \\
\frac{1}{\Gamma(7 / 3)}\left\{\frac{s(1-s)^{4 / 3}}{\left(4-6 s+4 s^{2}-s^{3}\right)^{1 / 3}}+\frac{2}{21 \sqrt[3]{3} \Delta}\left[2(1-s)^{4 / 3}+(2-2 s)^{4 / 3}\right]\right\}, 2 / 3 \leq s \leq 1, \\
J_{2}(s)=\frac{16}{3 \sqrt{\pi} \Delta}\left\{\frac{1}{7}(1-s)^{3 / 2}-\frac{1}{7}(1-s)^{7 / 2}-\frac{1}{5} s(1-s)^{5 / 2}\right\}, s \in[0,1],
\end{array}\right.
$$




$$
\begin{gathered}
J_{3}(s)=\frac{4}{3 \sqrt{\pi}}\left\{\frac{s(1-s)^{3 / 2}}{\left(3-3 s+s^{2}\right)^{1 / 2}}+\frac{4(1+\sqrt[3]{2})}{3 \sqrt[3]{3} \Delta}\left[\frac{1}{7}(1-s)^{3 / 2}-\frac{1}{7}(1-s)^{7 / 2}-\frac{1}{5} s(1-s)^{5 / 2}\right]\right\}, \quad s \in[0,1] \\
J_{4}(s)=\left\{\begin{array}{l}
\left.\frac{1}{6 \sqrt[3]{3} \Delta \Gamma(7 / 3)}\left[2(1-s)^{4 / 3}-2(1-3 s)^{4 / 3}+(2-2 s)^{4 / 3}-(2-3 s)^{4 / 3}\right)\right], 0 \leq s<1 / 3, \\
\frac{1}{6 \sqrt[3]{3} \Delta \Gamma(7 / 3)}\left[2(1-s)^{4 / 3}+(2-2 s)^{4 / 3}-(2-3 s)^{4 / 3}\right], 1 / 3 \leq s<2 / 3, \\
\frac{1}{6 \sqrt[3]{3} \Delta \Gamma(7 / 3)}\left[2(1-s)^{4 / 3}+(2-2 s)^{4 / 3}\right], 2 / 3 \leq s \leq 1 .
\end{array}\right.
\end{gathered}
$$

Then we deduce that assumptions $\left(J_{1}\right),\left(J_{2}\right)$ and $\left(J_{4}\right)$ are satisfied. In addition, by using the above functions $J_{i}$, $i=1, \cdots, 4$, we obtain $A:=\int_{0}^{1} J_{1}(s) \mathrm{d} s \approx 0.15972386, \quad B:=\int_{0}^{1} J_{2}(s) \mathrm{d} s \approx 0.05446581$, $C:=\int_{0}^{1} J_{3}(s) \mathrm{d} s \approx 0.09198682, \quad D:=\int_{0}^{1} J_{4}(s) \mathrm{d} s \approx 0.12885992$, and then $L=\max \{A+B, C+D\} \approx 0.22084674$. We choose $c_{0}=1$ and if we select $\tilde{a}<\frac{1}{L}, \tilde{b}<\frac{1}{L}$, then we conclude that $f(x)<\frac{1}{L}, g(x)<\frac{1}{L}$ for all $x \in[0,1]$. For example, if $\tilde{a} \leq 4.52$ and $\tilde{b} \leq 4.52$, then the above conditions for $f$ and $g$ are satisfied. So, assumption $\left(J_{3}\right)$ is also satisfied. By Theorems 2 and 3 we deduce that problem $\left(S_{0}\right)-\left(B C_{0}\right)$ has at least one positive solution for sufficiently small $a_{0}>0$ and $b_{0}>0$, and no positive solution for sufficiently large $a_{0}$ and $b_{0}$.

\section{Acknowledgements}

The work of R. Luca and A. Tudorache was supported by a grant of the Romanian National Authority for Scientific Research, CNCS-UEFISCDI, project number PN-II-ID-PCE-2011-3-0557.

\section{References}

[1] Baleanu, D., Diethelm, K., Scalas, E. and Trujillo, J.J. (2012) Fractional Calculus Models and Numerical Methods. Series on Complexity, Nonlinearity and Chaos. World Scientific, Boston.

[2] Das, S. (2008) Functional Fractional Calculus for System Identification and Control. Springer, New York.

[3] Kilbas, A.A., Srivastava, H.M. and Trujillo, J.J. (2006) Theory and Applications of Fractional Differential Equations. North-Holland Mathematics Studies, Elsevier Science, Amsterdam.

[4] Podlubny, I. (1999) Fractional Differential Equations. Academic Press, San Diego.

[5] Sabatier, J., Agrawal, O.P. and Machado, J.A.T., Eds. (2007) Advances in Fractional Calculus: Theoretical Developments and Applications in Physics and Engineering. Springer, Dordrecht.

[6] Samko, S.G., Kilbas, A.A. and Marichev, O.I. (1993) Fractional Integrals and Derivatives. Theory and Applications. Gordon and Breach, Yverdon.

[7] Agarwal, R.P., Andrade, B. and Cuevas, C. (2010) Weighted Pseudo-Almost Periodic Solutions of a Class of Semilinear Fractional Differential Equations. Nonlinear Analysis, Real World Applications, 11, 3532-3554. http://dx.doi.org/10.1016/j.nonrwa.2010.01.002

[8] Agarwal, R.P., Zhou, Y. and He, Y. (2010) Existence of Fractional Neutral Functional Differential Equations. Computers and Mathematics with Applications, 59, 1095-1100. http://dx.doi.org/10.1016/j.camwa.2009.05.010

[9] Aghajani, A., Jalilian, Y. and Trujillo, J.J. (2012) On the Existence of Solutions of Fractional Integro-Differential Equations. Fractional Calculus and Applied Analysis, 15, 44-69. http://dx.doi.org/10.2478/s13540-012-0005-4

[10] Ahmad, B. and Ntouyas, S.K. (2012) Nonlinear Fractional Differential Equations and Inclusions of Arbitrary Order and Multi-Strip Boundary Conditions. Electronic Journal of Differential Equations, 2012, 1-22. http://dx.doi.org/10.14232/ejqtde.2012.1.93

[11] Ahmad, B. and Ntouyas, S.K. (2012) A Note on Fractional Differential Equations with Fractional Separated Boundary Conditions. Abstract and Applied Analysis, 2012, Article ID: 818703. 
[12] Bai, Z. (2010) On Positive Solutions of a Nonlocal Fractional Boundary Value Problem. Nonlinear Analysis, 72, 916-924. http://dx.doi.org/10.1016/j.na.2009.07.033

[13] Balachandran, K. and Trujillo, J.J. (2010) The Nonlocal Cauchy Problem for Nonlinear Fractional Integrodifferential Equations in Banach Spaces. Nonlinear Analysis, 72, 4587-4593. http://dx.doi.org/10.1016/j.na.2010.02.035

[14] El-Shahed, M. and Nieto, J.J. (2010) Nontrivial Solutions for a Nonlinear Multi-Point Boundary Value Problem of Fractional Order. Computers and Mathematics with Applications, 59, 3438-3443. http://dx.doi.org/10.1016/j.camwa.2010.03.031

[15] Graef, J.R., Kong, L., Kong, Q. and Wang, M. (2012) Uniqueness of Positive Solutions of Fractional Boundary Value Problems with Non-Homogeneous Integral Boundary Conditions. Fractional Calculus and Applied Analysis, 15, 509528. http://dx.doi.org/10.2478/s13540-012-0036-x

[16] Jiang, D. and Yuan, C. (2010) The Positive Properties of the Green Function for Dirichlet-Type Boundary Value Problems of Nonlinear Fractional Differential Equations and Its Application. Nonlinear Analysis, 72, 710-719. http://dx.doi.org/10.1016/j.na.2009.07.012

[17] Liang, S. and Zhang, J. (2009) Positive Solutions for Boundary Value Problems of Nonlinear Fractional Differential Equation. Nonlinear Analysis, 71, 5545-5550. http://dx.doi.org/10.1016/j.na.2009.04.045

[18] Yuan, C. (2010) Multiple Positive Solutions for $(n-1,1)$-Type Semipositone Conjugate Boundary Value Problems of Nonlinear Fractional Differential Equations. Electronic Journal of Qualitative Theory of Differential Equations, 2010, 1-12. http://dx.doi.org/10.14232/ejqtde.2010.1.36

[19] Yuan, C., Jiang, D., O’Regan, D. and Agarwal, R.P. (2012) Multiple Positive Solutions to Systems of Nonlinear Semipositone Fractional Differential Equations with Coupled Boundary Conditions. Electronic Journal of Qualitative Theory of Differential Equations, 2012, 1-17. http://dx.doi.org/10.14232/ejqtde.2012.1.13

[20] Henderson, J. and Luca, R. (2014) Positive Solutions for a System of Fractional Differential Equations with Coupled integral Boundary Conditions. Applied Mathematics and Computation, 249, 182-197. http://dx.doi.org/10.1016/j.amc.2014.10.028

[21] Henderson, J., Luca, R. and Tudorache, A. (2015) On a System of Fractional Differential Equations with Coupled integral Boundary Conditions. Fractional Calculus and Applied Analysis, 18, 361-386. http://dx.doi.org/10.1515/fca-2015-0024

[22] Henderson, J. and Luca, R. (2013) Positive Solutions for a System of Nonlocal Fractional Boundary Value Problems. Fractional Calculus and Applied Analysis, 16, 985-1008. http://dx.doi.org/10.2478/s13540-013-0061-4

[23] Henderson, J. and Luca, R. (2014) Existence and Multiplicity of Positive Solutions for a System of Fractional Boundary Value Problems. Boundary Value Problems, 2014, 60. http://dx.doi.org/10.1186/1687-2770-2014-60

[24] Henderson, J., Luca, R. and Tudorache, A. (2015) Positive Solutions for a Fractional Boundary Value Problem. Nonlinear Studies, 22, 1-13.

[25] Luca, R. and Tudorache, A. (2014) Positive Solutions to a System of Semipositone Fractional Boundary Value Problems. Advances in Difference Equations, 2014, 179. http://dx.doi.org/10.1186/1687-1847-2014-179

[26] Henderson, J. and Luca, R. (2015) Boundary Value Problems for Systems of Differential, Difference and Fractional Equations: Positive Solutions. Elsevier, Amsterdam. 